\title{
TRICANONICAL SYSTEM OF A SURFACE OF GENERAL TYPE IN POSITIVE CHARACTERISTIC
}

\author{
TOHRU NAKASHIMA
}

(Communicated by Louis J. Ratliff, Jr.)

\begin{abstract}
Using vector bundle method, we study the tricanonical system on a minimal surface of general type defined over an algebraically closed field of positive characteristic. Under some conditions, it is proved that it has no fixed component.
\end{abstract}

\section{INTRODUCTION}

Pluricanonical systems on minimal surfaces of general type have been studied by many authors after the fundamental work of Bombieri [B]. Recently, Ekedahl extended many of the classical results of Bombieri to the positive characteristic case [E]. In particular, he proved that the $m$ th pluricanonical system $|m K|$ is base point free if $m \geq 4$ or $m \geq 3$ and $K^{2} \geq 2$. Some of the remaining cases are treated in $[\mathrm{SB}]$. In this short note we shall consider the fixed part of the tricanonical system $|3 K|$ under certain conditions. Our purpose is to show the following

Theorem. Let $X$ be a minimal surface of general type defined over an algebraically closed field $k$. Assume $\operatorname{char}(k)=p>2$ and $K^{2}=\chi\left(\mathscr{O}_{X}\right)=1$. Then $|3 K|$ has no fixed component if it is not composed with a pencil.

\section{PROOF OF THE THEOREM}

Proposition 1.1. Let $X$ be a minimal surface of general type defined over an algebraically closed field of characteristic $p>2$ such that $K^{2}=1$. Then $(-2)$ curves cannot be contained in the fixed part of $|3 K|$.

Proof. Suppose that a $(-2)$ curve $C$ is a fixed component of $|3 K|$. We have the exact sequence

$$
0 \rightarrow \mathscr{O}_{X}(3 K-C) \rightarrow \mathscr{O}_{X}(3 K) \rightarrow \mathscr{O}_{C}(3 K) \rightarrow 0,
$$

which induces a sequence

$$
H^{0}\left(\mathscr{O}_{X}(3 K)\right) \rightarrow H^{0}\left(\mathscr{O}_{C}(3 K)\right) \rightarrow H^{1}\left(\mathscr{O}_{X}(3 K-C)\right) \rightarrow H^{1}\left(\mathscr{O}_{X}(3 K)\right) .
$$

Received by the editors March 13, 1991 and, in revised form, May 17, 1991.

1991 Mathematics Subject Classification. Primary 14J29; Secondary $14 J 60$. 
Since $H^{1}\left(\mathscr{O}_{X}(3 K)\right) \cong H^{1}\left(\mathscr{O}_{X}(-2 K)\right)=0$ by [E, Theorem 1.7], our assumption implies that the map $H^{0}\left(\mathscr{O}_{X}(3 K)\right) \rightarrow H^{0}\left(\mathscr{O}_{C}(3 K)\right)$ is not surjective. Thus we have $H^{1}\left(\mathscr{O}_{X}(3 K-C)\right) \cong H^{1}\left(\mathscr{O}_{X}(C-2 K)\right) \neq 0$. Then there is a nonsplit extension

$$
0 \rightarrow \mathscr{O}_{X} \rightarrow E \rightarrow \mathscr{O}_{X}(2 K-C) \rightarrow 0
$$

where $E$ is a rank 2 vector bundle. $E$ satisfies the inequality $c_{1}(E)^{2}=$ $(2 K-C)^{2}=4>0=4 c_{2}(E)$.

By Theorem 1 in [SB], there exists a Frobenius map $F^{e}: X \rightarrow X$ such that the pullback $\left(F^{e}\right)^{*} E$ is unstable in the sense of Bogomolov. Therefore, we obtain an exact sequence

$$
0 \rightarrow \mathscr{O}_{X}\left(p^{e}(2 K-C)-\Delta\right) \rightarrow\left(F^{e}\right)^{*} E \rightarrow \mathscr{I}_{A} \otimes \mathscr{O}_{X}(\Delta) \rightarrow 0 .
$$

Here $\Delta$ is an effective divisor such that $p^{e}(2 K-C)-2 \Delta$ is contained in the positive cone of $X$ and $A$ is a 0 -dimensional subscheme (cf. [SB]). We shall consider the cases $e=0$ and $e>0$ separately and derive a contradiction.

(i) The case $e=0$. First we note, in this case, $\Delta \neq 0$. For, if $\Delta=0$ then $\operatorname{deg} A=0$ and our original sequence must split, which is a contradiction. Since $0<(2 K-C-2 \Delta) \cdot K=2-2 \Delta \cdot K$, we have $\Delta \cdot K=0$. Thus $\Delta$ is a sum of $(-2)$ curves and $\Delta^{2} \leq-2$. On the other hand, $\operatorname{deg} A=\Delta \cdot(C+\Delta-2 K)=$ $\Delta \cdot(C+\Delta) \geq 0$, so $\Delta \cdot C \geq-\Delta^{2} \geq 2$. Then

$$
\begin{aligned}
-2 & \geq(C+\Delta)^{2}=\Delta \cdot(C+\Delta)+\Delta \cdot C+C^{2} \\
& \geq \Delta \cdot(C+\Delta)+2-2=\Delta \cdot(C+\Delta) \geq 0 .
\end{aligned}
$$

This is a contradiction.

(ii) The case $e>0$. As in (i), $\left(p^{e}(2 K-C)-2 \Delta\right) \cdot K>0$ and thus we have $\Delta \cdot K \leq p^{e}-1$. According to [SB], there exists a purely inseparable covering $\rho: Y \rightarrow X$. We denote by $\omega_{Y}$ its dualizing sheaf. Then we compute, as in $[\mathrm{SB}]$,

$$
\begin{aligned}
\omega_{Y} \cdot \rho^{*} K & =2\left(p^{e}-1\right) K \cdot \Delta+p^{e}\left(K^{2}-\left(p^{e}-1\right)(2 K-C) \cdot K\right) \\
& \leq 2\left(p^{e}-1\right)^{2}+p^{e}\left(3-2 p^{e}\right)=2-p^{e}<0 .
\end{aligned}
$$

This implies that $Y$ is ruled. Let $q=q(X)$ be the irregularity of $X$. By Lemma 34 of $[\mathrm{SB}], \chi\left(\mathscr{O}_{Y}\right) \leq 1-q$. Furthermore, we have the inequality

$$
\chi\left(\mathscr{O}_{Y}\right) \geq p^{e}\left\{\chi\left(\mathscr{O}_{X}\right)+\frac{p^{e}-1}{12}\left[\left(2 p^{e}-1\right)(2 K-C)^{2}-3(2 K-C) \cdot K\right]\right\} .
$$

Since Corollary 1.8 of [E] gives $\chi\left(\mathscr{O}_{X}\right) \geq 1$, the above inequality shows $\chi\left(\mathscr{O}_{Y}\right)>$ 1 , which is impossible.

Lemma 1.2. Suppose $K^{2}=\chi\left(\mathscr{O}_{X}\right)=1$ and $\operatorname{char}(k)=p>2$. Then for an effective divisor $D$ that is numerically equivalent to $K$ (resp. $2 K$ ), we have $h^{0}(D) \leq 1$ (resp. $\left.h^{0}(D)=2\right)$.

Proof. Assume $D$ is numerically equivalent to $K$ and $h^{0}(D) \geq 2$. Then a Clifford argument shows $h^{0}(2 D) \geq 3$. Since $K$ is nef and big, $h^{2}(2 D)=$ $h^{0}(K-2 D)=h^{0}(-K)=0$. Moreover, by Corollary 22 of [SB] we have 
$h^{1}(2 D)=h^{1}(-(2 D-K))=0$. Therefore, the Riemann-Roch formula gives

$$
\begin{aligned}
h^{0}(2 D) & =\chi(2 D)=\frac{2 D \cdot(2 D-K)}{2}+\chi\left(\mathscr{O}_{X}\right) \\
& =\frac{2 K \cdot(2 K-K)}{2}+1=2 .
\end{aligned}
$$

Thus we have a contradiction. The second claim can be proved similarly.

Lemma 1.3. Let $X$ be as before. If $D$ is an effective divisor such that $h^{0}(D) \geq 2$ then $K \cdot D \geq 2$.

Proof. It is clear that $K \cdot D>0$. Suppose that $K \cdot D=1$. Then $K \cdot(K-D)=0$, and hence $D$ is numerically equivalent to $K$ or $(K-D)^{2}<0$. Since the former case is impossible by Lemma 1.2 , we have $(K-D)^{2}=1-2 K D+D^{2}<0$ and the result follows easily from this.

Using the preceding results we shall prove the theorem. Let $|3 K|=|M|+$ $F$ where $M$ is the moving part and $F$ the fixed part. Then $3=3 K^{2}=$ $M \cdot K+F \cdot K$. By Proposition 1.1 , we have $F \cdot K>0$ and by Lemma 1.3, $M \cdot K \geq 2$. Thus $F \cdot K=1$ and $M \cdot K=2$, and hence $K \cdot(M-2 K)=0$. Then we have $M^{2} \leq 3$ since Lemma 1.2 implies $(M-2 K)^{2}<0$. Let $\phi_{|M|}$ be the map associated to $|M|$. By assumption $|M|$ is not composed with a pencil, so $\operatorname{deg} \phi_{|M|} \cdot \operatorname{deg} \phi_{|M|}(X) \leq M^{2} \leq 3$. Since $h^{0}(3 K)=4, \phi_{|M|}(X)$ is a nondegenerate surface in $\mathbf{P}^{3}$. Then $\operatorname{deg} \phi_{|M|}(X) \geq 2$, and we obtain an obvious contradiction.

\section{REFERENCES}

[B] E. Bombieri, Canonical models of surfaces of general type, Inst. Hautes Études Sci. Publ. Math. 42 (1973), 171-220.

[E] T. Ekedahl, Canonical models of surfaces of general type in positive characteristic, Inst. Hautes Études Sci. Publ. Math. 67 (1988), 97-144.

[M] Y. Miyaoka, Tricanonical map of numerical Godeaux surfaces, Invent. Math. 34 (1976), 99-111.

[SB] N. I. Shepherd-Barron, Unstable vector bundles and linear systems on surfaces in characteristic $p$, preprint.

Department of Mathematics, Faculty of Science, Kyoto University, Kyoto, 606 Japan

Current address: Department of Mathematics, Faculty of Science, Tokyo Metropolitan University, Minami-Ohsawa 1-1, Hachioji-shi, Tokyo, 192-03 Japan 